\title{
Surface terms as counterterms in the AdS-CFT correspondence
}

\author{
Roberto Emparan* \\ Department of Mathematical Sciences, University of Durham, DH1 3LE, United Kingdom \\ and Departamento de Física Teórica, Universidad del País Vasco, Apdo. 644, E-48080 Bilbao, Spain \\ Clifford V. Johnson ${ }^{\dagger}$ \\ Department of Physics and Astronomy, University of Kentucky, Lexington, Kentucky 40506-0055 \\ Robert C. Myers \\ Physics Department, McGill University, Montréal, PQ, H3A 2T8, Canada \\ (Received 7 April 1999; published 1 October 1999)
}

\begin{abstract}
We examine the recently proposed technique of adding boundary counterterms to the gravitational action for spacetimes which are locally asymptotic to anti-de Sitter spacetimes. In particular, we explicitly identify higher order counterterms, which allow us to consider spacetimes of dimensions $d \leqslant 7$. As the counterterms eliminate the need of "background subtraction" in calculating the action, we apply this technique to study examples where the appropriate background was ambiguous or unknown: topological black holes, Taub-NUTAdS and Taub-Bolt-AdS. We also identify certain cases where the covariant counterterms fail to render the action finite, and we comment on the dual field theory interpretation of this result. In some examples, the case of a vanishing cosmological constant may be recovered in a limit, which allows us to check results and resolve ambiguities in certain asymptotically flat spacetime computations in the literature. [S0556-2821(99)07318-X]
\end{abstract}

PACS number(s): 04.62.+v, 04.65.+e, 11.10.Kk, 11.25.- $\mathrm{w}$

\section{INTRODUCTION}

The anti-de Sitter (AdS)-conformal field theory (CFT) correspondence asserts that there is an equivalence between a gravitational theory in $d$-dimensional anti-de Sitter spacetime and a conformal field theory residing in a $(d-1)$-dimensional "boundary" spacetime [1]. This equivalence or duality is best understood in the context of string theory with $d=5$, where the duality relates type IIB superstring theory on $\mathrm{AdS}_{5} \times \mathrm{S}^{5}$, and $\mathcal{N}=4$ supersymmetric Yang-Mills theory with gauge group $S U(N)$ in four dimensions $[2,3]$. The precise formulation of the AdS-CFT correspondence is made by equating the partition functions of the two theories:

$$
Z_{A d S}\left(\phi_{0, i}\right)=Z_{C F T}\left(\phi_{0, i}\right) .
$$

Here the fields $\phi_{0, i}$ have two interpretations: On the gravity side, these fields correspond to the boundary data or boundary values (up to a certain rescaling [1]) for the bulk fields $\phi_{i}$ which propagate in the AdS space. On the field theory side, these fields correspond to external source currents coupled to various CFT operators. Thus correlation functions of the operators in the CFT can be determined through a calculation using the dynamics of gravity in $\mathrm{AdS}$ spacetime $[1,3]$. In certain instances, one can consider evaluating the AdS partition function in a saddle-point approximation:

$$
e^{-I_{A d S}\left(\phi_{i}\right)}=\left\langle e^{\int \phi_{0, i} \mathcal{O}^{i}}\right\rangle_{C F T}
$$

\footnotetext{
*Email address: roberto.emparan@durham.ac.uk

†Email address: cvj@pa.uky.edu

${ }^{\ddagger}$ Email address: cm@hep.physics.mcgill.ca
}

where $I_{A d S}\left(\phi_{i}\right)$ is the classical gravitational action as a functional of the (super)gravity fields, and $\mathcal{O}^{i}$ are the dual CFT operators. Hence in this approximation, the AdS action becomes the generating function of the connected correlation functions in the CFT $[1,3]$. This framework is also naturally extended to considering CFT states for which certain operators acquire expectation values by considering solutions of the gravitational equations which are only asymptotically AdS [4,5].

One aspect of the duality which will be interesting for the present investigation is the choice of the background metric $\gamma_{i j}$ required to define the field theory. This metric is related by an infinite conformal transformation to the induced metric $h_{i j}$ on the boundary of the AdS spacetime [1]. Since the boundary conformal transformation is divergent, one regularizes the calculation by considering the induced metric for a family of surfaces which approach the boundary in a limit. This regularization procedure then will depend on the choices of coordinates in the asymptotic AdS region; i.e., it depends on the precise family of surfaces chosen. With different choices, the background geometry inherited by the CFT takes a completely different form. For example, depending on the choice of radial slicing for $\mathrm{AdS}_{n+1}$, the boundary geometry can be $S^{1} \times S^{n}, S^{n+1}, S^{1} \times \mathbb{R}^{n}$. We will discuss these and other possibilities in Sec. II. This procedure therefore allows one to study the CFT with different background geometries. From the point of view of the gravity theory, this procedure is interesting because naively the expressions on the left-hand side of Eqs. (1) and (2) are coordinate invariant. However, the asymptotic regularization explicitly breaks this covariance.

Returning to Eq. (2), considering the gravitational path integral in the saddle-point approximation has a long history in the quantum gravity literature, in particular in context of 
black hole thermodynamics [6] — see Refs. [7] and [8] for discussions relevant for AdS. There is a problem that must be faced with this approach in that typically the gravity action diverges. For $d=n+1$ spacetime dimensions, the familiar (Euclidean) action has two contributions

$$
\begin{aligned}
I_{\text {bulk }}+I_{\text {surf }}= & -\frac{1}{16 \pi G} \int_{\mathcal{M}} d^{n+1} x \sqrt{g}\left(R+\frac{n(n-1)}{l^{2}}\right) \\
& -\frac{1}{8 \pi G} \int_{\partial \mathcal{M}} d^{n} x \sqrt{h} K .
\end{aligned}
$$

The first term is just the Einstein-Hilbert-anti-de Sitter action with cosmological constant $\Lambda=-n(n-1) / 2 l^{2}$. The second integral is the Gibbons-Hawking boundary term which is required so that upon variation with metric fixed at the boundary, the action yields the Einstein equations [6]. Here, $K$ is the trace of the extrinsic curvature of the boundary $\partial \mathcal{M}$ as embedded in $\mathcal{M}$. In the AdS context, both of these expressions are divergent because the volumes of both $\mathcal{M}$ and $\partial \mathcal{M}$ are infinite (and the integrands are nonzero). The traditional approach to circumventing this problem is to perform a "background subtraction." That is, one produces a finite result by subtracting from Eq. (3) the contribution of a background reference spacetime, so that one can compare the properties of the solution of interest relative to those of the reference state. Note, however, that this subtraction requires that the asymptotic boundary geometries of the two solutions can be matched in order to render the surface contribution finite. ${ }^{1}$ Aside from being a technical nuisance, there are certain cases where an appropriate reference solution is ambiguous or unknown, e.g., topological black holes [9-13] and Taub-NUT (-Newman-Unit-Tamburino-)-AdS and Taubbolt-AdS $[14,15]$ - see discussion below.

In the context of the AdS-CFT correspondence, there does not seem to be room for a background subtraction in, for example, Eq. (2). Remarkably AdS spacetime offers an alternative approach. The divergences that arise in Eq. (3) are all proportional to local integrals of the background CFT metric $\gamma_{i j}[1,16]$. Thus these divergences can be eliminated by extending the regularization procedure for the action with a "counterterm subtraction." That is Eq. (3) is modified to include the subtraction of a finite set of boundary integrals (with divergent coefficients) involving curvature scalars constructed from the background metric $\gamma_{i j}$ [17]. Recently a remarkable insight was provided by Ref. [18] (see also Ref. [19]): If the counterterms are expressed in terms of the induced metric $h_{i j}$, rather than $\gamma_{i j}$, then they naturally appear with the appropriate divergences, as the volume of the regulator surface grows as it approaches the boundary of AdS spacetime. Thus in the counterterm subtraction approach, one may produce a finite gravitational action by supplementing the contributions in Eq. (3) with an extra surface integral

\footnotetext{
${ }^{1}$ Again, there is the implicit need for a regularization procedure with regards to the asymptotic boundary.
}

$$
I_{\mathrm{ct}}=\frac{1}{8 \pi G} \int_{\partial \mathcal{M}} d^{n} x \sqrt{h} F(l, \mathcal{R}, \nabla \mathcal{R}),
$$

where the counterterms depend only on the curvature $\mathcal{R}$ (and its derivatives) of the induced boundary metric $h_{i j}$ - see Sec. III for explicit expressions. That this construction is unique to asymptotically $\mathrm{AdS}$ spaces is apparent because the AdS curvature scale $l$ is essential in defining the counterterms. ${ }^{2}$ Note that these expressions are universal depending only on $l$ and the spacetime dimension. Once these are fixed, one may use the same counterterms to regulate the action for any choice of coordinates on any asymptotically AdS solution. ${ }^{3}$

Even outside of the AdS-CFT correspondence, counterterm subtraction provides a remarkable new theoretical tool with which to investigate gravitational physics. Together, Eqs. (3) and (4) provide a finite covariant definition of the gravitational action for asymptotically $\mathrm{AdS}$ spaces. As a simple example, one might consider the energy of a gravitating system in AdS space. Traditionally the definition of energy in gravity required a background reference solution in asymptotically AdS spaces [8,21], just as in asymptotically flat spaces [22]. Combined with the quasilocal formulation of Brown and York [23], the AdS action with counterterms provides a definition of energy that is independent of any reference solution [18]. Using this technique, one discovers a finite energy for $\mathrm{AdS}_{5}$ with an $\mathbb{R} \times S^{3}$ boundary. In the context of the AdS-CFT correspondence, one can interpret this energy as the Casimir energy of the dual field theory in the latter background geometry [18]. A similar Casimir energy arises in $\mathrm{AdS}_{3}$ [18], where there is a well-known difference between the energy $M=-1 /(8 G)$ of global $\mathrm{AdS}_{3}$ and that of the $M=0$ state (which is only locally $\mathrm{AdS}_{3}$ ).

Thus one might revisit Euclidean quantum gravity with this new theoretical tool in hand. In particular, one can address the cases where the background subtraction technique was not possible or (due to ambiguities) the results were disputed. This is one of the primary objectives of the current investigation.

The issue of the correct reference state has been disputed for "topological black holes" [9-13], in particular for the 'hyperbolic AdS black holes.' The latter are black hole solutions where the horizon is a hyperbolic space $H^{n}$ instead of a sphere. As it happens, there is among these solutions one which is locally (though not globally) equal to AdS. However, in order for it to be regular, the Euclidean time has to take a fixed finite value - in other words, it is a finite temperature solution. As such, it is not an adequate reference state for matching calculations, which would require a solution that admits arbitrary Euclidean period. In Sec. IV, we apply the counterterm subtraction prescription to compute

\footnotetext{
${ }^{2} \mathrm{We}$ are excluding non-polynomial terms, which could be introduced in the absence of a cosmological constant [20].

${ }^{3}$ Actually this is not quite the complete story — see below and Sec. VI.
} 
the action, and discover some intriguing results. We are led to speculate on a connection to the "precusor" states recently discussed in Ref. [33].

The Taub-NUT solution is known to admit an extension to include a cosmological constant [24], and as such, the Taub-NUT-anti-de Sitter (TN-AdS) solution has been studied recently in Refs. [14,15]. The boundary geometry cannot be matched to that of AdS space, and so there is no known reference solution with which to make a background subtraction. Instead, in Refs. [14,15], the analog of the self-dual TN solution (i.e., the one with a "nut," a zero-dimensional fixed point set, at its origin) was used as the reference state in a background subtraction calculation of the action of the Taub-bolt-anti-de Sitter (TB-AdS) solution. In Sec. V, we use the counterterm subtraction for a backgroundless calculation of the action of TN-AdS. This allows us to study the thermodynamics of this solution in and of itself. In particular, we can study its local intrinsic stability, and find its entropy, as a function of the nut charge. This leads to some surprises.

As mentioned above, the counterterm subtraction approach cannot be extended in a straightforward way to asymptotically flat $(\mathrm{AF})$ spacetimes (and for that matter, to spacetimes which do not asymptote to AdS) because the AdS scale is an essential ingredient in the definition of the counterterms (4). However, one can apply this technique in a case-by-case manner to the computation of the action of those asymptotically flat solutions which can be obtained as limits of AdS solutions. A simple example is the computation of the action of the Schwarzschild solution by first embedding it in AdS spacetime. There exists a SchwarzschildAdS solution [7] - discussed extensively in the context of the AdS-CFT correspondence recently [1,4] — which for black holes that are much smaller than the cosmological length scale $l \sim|\Lambda|^{-1 / 2}$ approximates the asymptotically flat Schwarzschild solution. We can compute the action of this Schwarzschild-AdS black hole by using the counterterm prescription, and then take the limit $l \rightarrow \infty$. In this way we almost recover the standard result that is obtained by matching the AF solution to Minkowski spacetime.

The preceding is a satisfying, but somewhat trivial example. However, there are other cases of AF spaces where the computation of the action, using the more traditional background subtraction technique, has been the subject of some controversy. One such case is that of the Taub-NUT solutions, which are only asymptotically locally flat (ALF). In Ref. [25] the action of generic Euclidean Taub-NUT solutions (of which only the self-dual Taub-NUT and Taubbolt instantons are regular) was computed by trying to match the solutions to Minkowski space, in order to perform a regularizing subtraction (a similar matching was also attempted in Ref. [26]). However, since the large radius slices of Euclidean Taub-NUT space are squashed three-spheres, in contrast to the Minkowskian slices $S^{1} \times S^{2}$, the matching is not really well defined. Therefore, it was proposed in Ref. [27] that the proper background to be subtracted is instead the self-dual Taub-NUT instanton, which has the lowest possible energy among the regular Euclidean Taub-NUT solutions- the only other regular solution is the Taub-bolt instanton. ${ }^{4}$ It was noticed in Ref. [14] that there existed a branch of solutions which tends to the ALF Taub-NUT solution as $l \rightarrow \infty$. (These are the analogue of the "small" Schwarzschild black hole branch of solutions on Ref. [7].) Therefore, after applying the counterterm subtraction procedure to compute the action of the asymptotically TN-AdS solution, we take the limit $l \rightarrow \infty$. This limit provides then a "background independent" result for the action of the ALF Taub-NUT solutions. Remarkably, we find that the result agrees precisely with the "imperfect matching" one given in Ref. [25]. Furthermore, we show that the counterterm prescription results are reproduced by performing an "imperfect matching" to AdS similar to the one in the ALF case.

A simple application of the counterterm subtraction is to calculate the action of (Euclidean) $\mathrm{AdS}_{n+1}$ for different choices of coordinates, i.e., with different boundary geometries. In Sec. VI, we present an analysis of the multi-slicing phenomenon for (Euclidean) $\operatorname{AdS}_{n+1}$ with $n \leqslant 4$, showing the results for the action in several different cases. It is interesting to note the appearance of different Casimir energies in the various cases. A more dramatic result is that for certain boundary geometries, such as $S^{n}$ and $H^{n}$, one finds that the counterterm subtraction is insufficient. That is, a divergence that is logarithmic in the asymptotic radius appears, and cannot be eliminated by the addition of a local counterterm as in Eq. (4). These divergences which can arise for even $n$ have been noted previously in the context of the AdS-CFT correspondence $[1,17]$. There they may be related to a conformal anomaly for the dual CFT in certain background geometries which is well known to be connected to the appearance of logarithmic divergences in the effective field theory action [28]. Of course, this presents a limitation on counterterm subtraction as a general tool to investigate asymptotically AdS spaces in odd spacetime dimensions.

Certainly our results have many interesting implications for the dual field theory via the AdS-CFT correspondence. We will only make limited comments on this aspect of the work here, leaving a more general study of the field theoretic interpretation for a future paper.

While this work was being completed, we were informed that Mann [29] had also considered the application of the AdS action with counterterms to the solutions considered in Sec. IV and V.

\section{MANY FACES OF AdS SPACETIME}

As described in the previous section, counterterm subtraction works by subtracting the integral of various boundary curvature invariants (4) from the standard action (3). This leaves unspecified the way in which the boundary of AdS spacetime is approached, i.e., the choice of "radial" coordinate defining the family of surfaces which approach the

\footnotetext{
${ }^{4}$ Some care should be exercised, since often in the literature the name "Taub-NUT solution" is used to refer specifically to the self-dual Taub-NUT instanton, instead of the full, two-parameter Taub-NUT solution.
} 
boundary as a limit. Depending on this choice, the slices at constant radius can have different geometry or even different topology. Even if the spaces are locally equivalent to one another, the computation of the action will in general lead to different results, since the boundary terms in the action will take different values. In the end, since all different forms of a spacetime will be related by diffeomorphisms, with possible addition or subtraction of points, and possibly as well, identifications under discrete subgroups, the different results for the action will bear a relation to one another, too. Here we will describe some of the many possible "faces" of the boundary of AdS spacetime. In subsequent sections, we will consider these metrics as examples for the application of the counterterm subtraction technique, and compare the results. Clearly, such a comparison would have been impossible had we required a background for the calculation.

Let us first present Euclidean $\operatorname{AdS}_{n+1}$ in the following three familiar metrics,

$$
d s^{2}=\left(k+\frac{r^{2}}{l^{2}}\right) d \tau^{2}+\frac{d r^{2}}{\left(k+r^{2} / l^{2}\right)}+\frac{r^{2}}{l^{2}} d \Sigma_{k, n-1}^{2},
$$

where the $(n-1)$-dimensional metric $d \Sigma_{k, n-1}^{2}$ is

$$
d \Sigma_{k, n-1}^{2}= \begin{cases}l^{2} d \Omega_{n-1}^{2} & \text { for } k=+1, \\ \sum_{i=1}^{n-1} d x_{i}^{2} & \text { for } k=0, \\ l^{2} d \Xi_{n-1}^{2} & \text { for } k=-1,\end{cases}
$$

where $d \Omega_{n-1}^{2}$ is the unit metric on $S^{n-1}$. By $H^{n-1}$ we mean the $(n-1)$-dimensional hyperbolic space, whose "unit metric" $d \Xi_{n-1}^{2}$ can be obtained by analytic continuation of that on $S^{n-1}$. It is straightforward to see that all of the above solutions are locally equivalent to each other. In the above we are assuming that $n>2$ for $k=-1$, since for $n=2$ one does not have a hyperbolic metric $H^{1}$.

For later use in the paper, we will write the volume of the space $d \Sigma_{k, n}^{2}$ as $l^{n} \sigma_{k, n}$. In this way, $\sigma_{k=+1, n}$ will be equal to the volume $\omega_{n}$ of the unit $n$-sphere.

Next we consider Euclidean $\operatorname{AdS}_{n+1}$ with metric

$$
d s^{2}=\frac{d r^{2}}{\left(k+r^{2} / l^{2}\right)}+\frac{r^{2}}{l^{2}} d \Sigma_{k, n}^{2},
$$

where the $n$-dimensional metric $d \Sigma_{k, n}^{2}$ is defined precisely in the same way as above in Eq. (6). For $k=0$, this simply reproduces the $k=0$ metric in Eq. (5). One might note that a transformation of the radial coordinate brings these metrics into the form

$$
d s^{2}=l^{2} d \rho^{2}+f_{k}^{2}(\rho) d \Sigma_{k, n}^{2},
$$

where

$$
f_{k}(\rho)= \begin{cases}\sinh \rho & \text { for } k=+1 \\ e^{\rho} & \text { for } k=0 \\ \cosh \rho & \text { for } k=-1\end{cases}
$$

One final AdS metric which we will consider is

$$
d s^{2}=\frac{d r^{2}}{\left(k+r^{2} / l^{2}\right)}+\left(k+\frac{r^{2}}{l^{2}}\right) d \hat{\Sigma}_{-k, \hat{m}}^{2}+\frac{r^{2}}{l^{2}} d \widetilde{\Sigma}_{k, \tilde{m}}^{2},
$$

where again the metrics $d \hat{\Sigma}_{-k, \hat{m}}^{2}$ and $d \widetilde{\Sigma}_{k, \tilde{m}}^{2}$ are defined in Eq. (6). For $k=0$ we once again reproduce the $k=0$ metric in Eq. (5). For $k= \pm 1$, we assume that both $\hat{m}, \tilde{m} \geqslant 2$. For $k=+1$, the boundary geometry is $H^{\hat{m}} \times S^{\tilde{m}}$, while for $k$ $=-1$, we simply interchange the hyperbolic space and the sphere. However, in the latter case, the coordinate transformation $\widetilde{r}^{2}=r^{2}-l^{2}$ puts the metric back in the $k=+1$ form with $\hat{m} \leftrightarrow \tilde{m}$.

Thus with the metrics in Eqs. (5), (7) and (10), we have displayed $\operatorname{AdS}_{n+1}$ with a wide variety of boundary geometries:

$$
\begin{gathered}
\mathbb{R}^{n}, S^{1} \times S^{n-1}, \quad S^{1} \times \mathbb{R}^{n-1}, S^{1} \times H^{n-1}, \\
S^{n}, \quad H^{n}, \quad S^{m} \times H^{n-m} .
\end{gathered}
$$

All of these AdS metrics are maximally symmetric, i.e.,

$$
R_{i j k l}=-\frac{1}{l^{2}}\left(g_{i k} g_{j l}-g_{i l} g_{j k}\right),
$$

which ensures that the geometry is conformally flat. This condition also ensures the geometries are all locally AdS.

It is interesting to notice the form of some of the boundary geometries we get here upon analytic continuation to Minkowski signature, since they are rather common:

$$
\begin{aligned}
& S^{n}: \text { Euclidean de Sitter space } \\
& H^{n}: \text { (global) anti-de Sitter space } \\
& \mathbb{R}^{n}: \text { Minkowski space. }
\end{aligned}
$$

Furthermore, if we assume a specific analytic continuation to Lorentzian spacetime, e.g., $S^{1} \times S^{n} \rightarrow \mathbb{R}($ time $) \times S^{n}$, then

$$
\begin{aligned}
& \mathbb{R} \times S^{n-1} \text { : the Einstein static universe } \\
& \mathbb{R} \times H^{n-1} \text { :the static open universe. }
\end{aligned}
$$

The AdS-CFT correspondence implies then an equivalence between, on the one hand, quantum gravity in AdS and, on the other hand, a CFT on any of the above backgrounds. We find it particularly amusing that, when the boundary is taken to be $H^{n}$, quantum gravity in $\operatorname{AdS}_{n+1}$ can be dual to a CFT on an $\operatorname{AdS}_{n}$ background. It should be kept in mind that the geometry on the boundary is not dynamical, since there are no gravitational degrees of freedom in the dual CFT.

There is an important feature that distinguishes the solutions with $k=-1$ from those with $k=0,+1$ : there is a finite minimum radius $r=l$ at which $g_{r r}$ diverges. In Eq. (5), the Killing vector $\partial_{\tau}$ also has fixed point set (a "bolt") at this radius. In this case, the Euclidean solution will be regular only if the coordinate $\tau$ is identified with period $\beta=2 \pi l$. In 
the metric (7) with $k=-1$, the minimum radius $r=r_{+}$simply denotes the boundary of a coordinate patch as is evident from the form of the metric in Eq. (8) with the new radial coordinate $\rho$. In the case of Eq. (10) with $k=-1, r=r_{+}$is the location of a "conical" singularity. For $k=+1$, the minimum radius is $r=r_{+}=0$ and the geometry is smooth at this point in metrics (5) and (7), but it corresponds to a "conical singularity" in Eq. (10). For $k=0$, the minimum radius is again $r=r_{+}=0$ which in this case is an infinite proper distance away and so there is no problem with the curvature here. Note that the geometries with conical singularities or a bolt are only locally AdS; that is, they describe AdS spacetime with additional discrete identifications of points.

Equation (12) is an extremely restrictive condition. If one is simply interested in solving Einstein's equations with a negative cosmological constant

$$
R_{i j}=-\frac{n-2}{l^{2}} g_{i j}
$$

then the above metrics remain solutions when the boundary geometries are replaced by arbitrary Einstein spaces. In all of the metrics (5), (7), (10), one may replace any of the $S^{p}$ factors (with $p>1)$ by a space satisfying $\widetilde{R}_{a b}=(p$ -1) $/ l^{2} \tilde{g}_{a b}$. Similarly any $H^{p}$ factors can be replaced by a space satisfying $\hat{R}_{a b}=-(p-1) / l^{2} \hat{g}_{a b}$, and $\mathbb{R}^{p}$ factors can be replaced by any Ricci flat solution, i.e., $R_{a b}=0$. For example, then $S^{p}$ can be replaced by a product of spheres $S^{p_{1}}$ $\times \cdots \times S^{p_{q}}$ where $\sum_{i=1}^{q} p_{i}=p$ with $p_{i}>2$ and the radii of the individual spheres is scaled so $r_{i}^{2}=(p-1) /\left(p_{i}-1\right) l^{2}$. These generalized solutions will no longer be conformally flat or locally AdS. Furthermore, generically a true curvature singularity is introduced at the minimum radius, e.g., $R_{i j k l} R^{i j k l}$ grows without bound as $r$ approaches $r_{+}$.

\section{COUNTERTERM ACTION}

The detailed form of the boundary counterterms was originally explored in Ref. [17], where they were derived in terms of the background (field theory) metric $\gamma_{i j}$. The insight provided by Ref. [18] was that the counterterms should be written in terms of the induced metric on the boundary $h_{i j}$. In this way, they naturally appear with the appropriate (infinite volume) divergences to cancel those arising from the classical gravitational action. The focus of Ref. [18] was to construct a finite boundary stress tensor without using a reference background. However, the proposed prescription naturally provides the construction of a finite action which can then be employed, for example, to calculate the action of Euclidean gravitational instantons. This will be the primary application which we consider in the following.

Hence the full (Euclidean) gravitational action in $d=n$ +1 spacetime dimensions has three contributions

$$
I_{\text {AdS }}=I_{\text {bulk }}\left(g_{i j}\right)+I_{\text {surf }}\left(g_{i j}\right)+I_{\mathrm{ct}}\left(h_{i j}\right) .
$$

The first two terms, comprising the familiar classical action, were given in Eq. (3). Here, $h_{i j}$ is the induced metric on the boundary $\partial \mathcal{M}$ which may be defined as $h_{i j}=g_{i j}-n_{i} n_{j}$ where $n$ is an outward pointing unit normal vector to $\partial \mathcal{M}$. In the Gibbons-Hawking boundary term $I_{\text {surf }}$, the trace of the extrinsic curvature is defined by $K=h^{i j} \nabla_{i} n_{j}{ }^{5}$

The counterterm action $I_{\mathrm{ct}}\left(h_{i j}\right)$ may be arranged as an expansion in powers of the boundary curvature (and its derivatives). The number of terms that appears grows with the dimension of the spacetime. The first few terms are explicitly

$$
\begin{aligned}
I_{\mathrm{ct}}= & \frac{1}{8 \pi G} \int_{\partial \mathcal{M}} d^{n} x \sqrt{h}\left[\frac{n-1}{l}+\frac{l}{2(n-2)} \mathcal{R}\right. \\
& \left.+\frac{l^{3}}{2(n-4)(n-2)^{2}}\left(\mathcal{R}_{a b} \mathcal{R}^{a b}-\frac{n}{4(n-1)} \mathcal{R}^{2}\right)+\cdots\right],
\end{aligned}
$$

where $\mathcal{R}$ and $\mathcal{R}_{a b}$ are the Ricci scalar and Ricci tensor for the boundary metric, respectively. Combined these three counterterms are sufficient ${ }^{6}$ to cancel divergences for $n \leqslant 6$. In this covariant form, the first term originally appeared in Ref. [19], ${ }^{7}$ while the second term first appeared in Ref. [18]. We derived the third term by demanding that the infinite volume divergences were cancelled when using the metric (10). Any of these terms may be derived with the construction provided by Ref. [17] for the appropriate curvature integral in terms of the CFT metric $\gamma_{i j}$. One then simply substitutes the induced boundary metric $h_{i j}$ to produce the covariant counterterms appearing in $I_{\mathrm{ct}}$. To go to higher dimensions, resorting to this construction seems inescapable as the "simple" asymptotically AdS metrics presented in Sec. II cannot be used to distinguish all of the curvature invariants that can appear in the higher order counterterms. It is important to note that the fact that we have counterterms for dimensions up to $d=7$ means that we can now study all (known) AdS applications which arise in string theory and M theory.

Other matter field actions, for example an action for Maxwell fields, can be added to Eq. (16). Although, at least for black hole solutions, the addition of gauge fields does not seem to require new counterterms [32], we must remain alert to the possibility that extra matter fields may require the addition of new, non-geometric surface counterterms to the action. This issue will not be considered further here.

As a simple example, we will consider calculating the action (16) with the metric (5) for AdS spacetime with boundary $S^{1} \times M_{k}$. Let us present the contributions of the individual terms in the action:

\footnotetext{
${ }^{5}$ Our conventions differ by signs from Refs. [18,23], but are chosen to conform with standard practice in general relativity, as in, e.g., Ref. [30].

${ }^{6}$ Or almost, see Sec. IV.

${ }^{7}$ This term had also been considered to provide a (partial) regularization of the action of $\mathrm{AdS}_{5}$ in Ref. [31].
} 


$$
\begin{aligned}
& I_{\text {bulk }}=\frac{\beta \sigma_{k, n-1}}{8 \pi G l^{2}}\left[-r_{+}^{n}+r^{n}\right], \\
& I_{\text {surf }}=\frac{\beta \sigma_{k, n-1}}{8 \pi G l^{2}}\left[r^{n}\left(-n-k(n-1) \frac{l^{2}}{r^{2}}\right)\right], \\
& I_{\mathrm{ct}}^{1}=\frac{\beta \sigma_{k, n-1}}{8 \pi G l^{2}}\left[r^{n}(n-1)\left(1+k \frac{l^{2}}{r^{2}}\right)^{1 / 2}\right] \\
& =\frac{\beta \sigma_{k, n-1}}{8 \pi G l^{2}}\left[r ^ { n } ( n - 1 ) \left(1+\frac{k}{2} \frac{l^{2}}{r^{2}}-\frac{k^{2}}{8} \frac{l^{4}}{r^{4}}\right.\right. \\
& \left.\left.+\frac{k^{3}}{16} \frac{l^{6}}{r^{6}}+\cdots\right)\right] \\
& I_{\mathrm{ct}}^{2}=\frac{\beta \sigma_{k, n-1}}{8 \pi G l^{2}}\left[r^{n}(n-1) \frac{k}{2} \frac{l^{2}}{r^{2}}\left(1+k \frac{l^{2}}{r^{2}}\right)^{1 / 2}\right] \\
& =\frac{\beta \sigma_{k, n-1}}{8 \pi G l^{2}}\left[r ^ { n } ( n - 1 ) \left(\frac{k}{2} \frac{l^{2}}{r^{2}}+\frac{k^{2}}{4} \frac{l^{4}}{r^{4}}\right.\right. \\
& \left.\left.-\frac{k^{3}}{16} \frac{l^{6}}{r^{6}}+\cdots\right)\right] \\
& I_{\mathrm{ct}}^{3}=\frac{\beta \sigma_{k, n-1}}{8 \pi G l^{2}}\left[r^{n}(n-1)\left(-\frac{k^{2}}{8} \frac{l^{4}}{r^{4}}\right)\left(1+k \frac{l^{2}}{r^{2}}\right)^{1 / 2}\right] \\
& =\frac{\beta \sigma_{k, n-1}}{8 \pi G l^{2}}\left[r^{n}(n-1)\left(-\frac{k^{2}}{8} \frac{l^{4}}{r^{4}}-\frac{k^{3}}{16} \frac{l^{6}}{r^{6}}+\cdots\right)\right],
\end{aligned}
$$

where $\sigma_{k, n-1}$ is the (dimensionless) volume of the space with metric $d \Sigma_{k, n-1}^{2} / l^{2}$, and $\beta$ is the period of $\tau$. We have also separated the contributions of the individual counterterms in Eq. (17), so $I_{\mathrm{ct}}^{i}$ is the integral of the $i$ th term in the action. Now, for a particular boundary dimension only some of the counterterms are included to cancel the divergences. So for $n=2 i-1,2 i$, one keeps only up to $I_{\mathrm{ct}}^{i}$. For any odd value of $n$, one has then

$$
I_{k, n+1}=-\frac{\beta \sigma_{k, n-1}}{8 \pi G l^{2}}\left[r_{+}^{n}+O\left(l^{n+1} / r\right)\right] .
$$

For the even values of $n$, an extra constant term makes an appearance so that

$$
\begin{aligned}
I_{k, n+1}= & \frac{\beta \sigma_{k, n-1}}{8 \pi G l^{2}}\left(-r_{+}^{n}-\frac{k}{2} l^{2} \delta_{n, 2}+\frac{3 k^{2}}{8} l^{4} \delta_{n, 4}-\frac{5 k}{16} l^{6} \delta_{n, 6}\right. \\
& \left.+\cdots+O\left(l^{n+1} / r\right)\right)
\end{aligned}
$$

As we have explained above, for $k=+1,0$, we have $\beta$ arbitrary and $r_{+}=0$, whereas for $k=-1, r_{+}=l$ and $\beta=2 \pi l$.
Note that for even $n$, the coefficients of the higher counterterms are actually divergent, even though they formally evaluate to a finite result. Further in either of these results, Eqs. (19) and (20), there are extra terms of order $1 / r$, which vanish when the limit $r \rightarrow \infty$ is taken in order to approach the AdS boundary. However, consider the case of $n$ odd, where we have in fact the option of keeping all of the higher order counterterms in Eq. (17), i.e., including the terms which actually vanish in the boundary limit. This would give a result where in fact all of the inverse powers $r^{-p}$ would be cancelled so that not only would the action be finite, but it would be independent of the regulator radius.

Given the explicit counterterms in Eq. (17), we can only really evaluate the action for $n \leqslant 6$. However, keeping in mind that the higher order counterterms ensure the cancellation of divergences order by order, it is clear that the formulas (19) and (20) will be unchanged for $n>6$. Further we can show that the coefficient of the extra contributions for $n$ even will be

$$
(-k)^{n / 2} \frac{(n-1) ! !^{2}}{n !} l^{n}
$$

To derive this result, note that the bulk and surface contributions can be written as

$$
I_{\mathrm{bulk}}+I_{\mathrm{surf}}=\frac{\beta \sigma_{k, n-1}}{8 \pi G l^{2}}\left[-r_{+}^{n}-(n-1) r^{n}(1+x)\right]
$$

where $x=k l^{2} / r^{2}$, while the counterterms yield

$$
I_{\mathrm{ct}}^{p}=\frac{\beta \sigma_{k, n-1}}{8 \pi G l^{2}}(n-1) r^{n} c_{p} x^{p-1}(1+x)^{1 / 2}
$$

where $c_{p}$ are constants independent of $n$. The key point is to realize that the counterterm contributions will cancel the $x$ dependence in Eq. (22) to an arbitrarily large order, and hence these coefficients are just the coefficients in the Taylor series

$$
(1+x)^{1 / 2}=\sum_{p=1}^{\infty} c_{p} x^{p-1}
$$

Now as stated above for a given $n=2 i$, the action only includes the finite sum: $I_{\text {bulk }}+I_{\text {surf }}+\sum_{p=1}^{i} I_{\mathrm{ct}}^{p}$. Thus with some elementary manipulations, one finds the residual finite term in Eq. (20) appears with the coefficient (21) above.

\section{AdS BLACK HOLES}

In this section we turn to the study of black hole solutions, using the counterterm subtraction scheme. In the presence of a negative cosmological constant, the horizon of a black hole admits a much larger variety of geometries and topologies than in asymptotically flat situations. This is consistent with the variety of boundary topologies that we can obtain for $\mathrm{AdS}$ itself, depending upon how we choose to radially foliate it, as discussed in Sec. II. The case ( $k=1$, below) of spherical black holes has already been studied using this counter- 
term subtraction scheme in Ref. [18], but we compute and list those results in what follows for completeness and for comparison with the flat and hyperbolic cases.

In Ref. [13], it was shown that the Einstein-anti-de Sitter system in $n+1$ dimensions admits the following solutions:

$$
d s^{2}=-V_{k}(r) d t^{2}+\frac{d r^{2}}{V_{k}(r)}+\frac{r^{2}}{l^{2}} d \Sigma_{k, n-1}^{2},
$$

with

$$
V_{k}(r)=k-\frac{\mu}{r^{n-2}}+\frac{r^{2}}{l^{2}},
$$

where the $(n-1)$ dimensional metric $d \Sigma_{k, n-1}^{2}$ is defined as in Eq. (6). Thus it represents $S^{n-1}, \mathbb{R}^{n-1}$ and $H^{n-1}$ for $k$ $=+1,0$ and -1 , respectively. A spacetime that is locally the same as anti-de Sitter is recovered when $\mu=0$ for which the metric reduces to that in Eq. (5).

By going to the Euclidean section one finds that the Euclidean time period (the inverse temperature) has to be

$$
\beta=\frac{4 \pi l^{2} r_{+}}{n r_{+}^{2}+k(n-2) l^{2}} .
$$

Here, $r_{+}$is the largest positive root of $V_{k}(r)$, typically associated with the outer horizon of a black hole. For $k=1$ and $\mu=0$ (global AdS spacetime), there is no such root, but the correct results are obtained by setting $r_{+}=0$. Now, it is important to notice that, whereas for $k=\{1,0\}$ the locally $\operatorname{AdS}$ solution corresponds to $r_{+}=0$, this is not true for $k=-1$. AdS spacetime with hyperbolic slicing has a bifurcate Killing horizon at $r=l$ and a fixed temperature $\beta=2 \pi l$. By contrast, there exists an extremal $k=-1$ solution, with a degenerate horizon at $r=r_{e}$ and parameter $\mu=\mu_{e}$, satisfying

$$
r_{e}=\sqrt{\frac{n-2}{n}} l, \quad \mu_{e}=-\frac{2}{n-2}\left(\frac{n-2}{n}\right)^{n / 2} l^{n-2} .
$$

In particular,

$$
\begin{aligned}
& \mu_{e}=-\frac{2 l}{3 \sqrt{3}}, \quad r_{e}=\frac{l}{\sqrt{3}}, \quad \text { for } n=3, \\
& \mu_{e}=-\frac{l^{2}}{4}, \quad r_{e}=\frac{l}{\sqrt{2}}, \quad \text { for } n=4, \\
& \mu_{e}=-\frac{4 l^{4}}{27}, \quad r_{e}=\sqrt{\frac{2}{3}} l, \quad \text { for } n=6 .
\end{aligned}
$$

Therefore, in a calculation for $k=-1$ of the action, with background matching, the question arises concerning which is the correct background to subtract: On the one hand, the locally AdS solution - which has the higher symmetrymight be physically appealing. However, since its period $\beta$ is fixed, matching it to a solution with a different value of $\beta$ would introduce a conical singularity at the horizon [11]. On the other hand, the extremal solution, with a lower value of $\mu$ (and as we will see, of the energy), has arbitrary $\beta$ and therefore can be matched to any other solution. Hence, the extremal solution was the preferred background for the matching calculations in Refs. [11-13].

It is clear from this discussion that the method of counterterm subtraction can be of help here. For the solutions described above we obtain

$$
\begin{aligned}
I_{k, n+1}= & \frac{\beta \sigma_{k, n-1}}{8 \pi G l^{2}}\left(-r_{+}^{n}+\frac{\mu l^{2}}{2}-\frac{k}{2} l^{2} \delta_{n, 2}\right. \\
& \left.+\frac{3 k^{2}}{8} l^{4} \delta_{n, 4}-\frac{5 k}{16} l^{6} \delta_{n, 6}+\cdots\right) \\
= & \frac{\beta \sigma_{k, n-1}}{16 \pi G l^{2}}\left(k r_{+}^{n-2} l^{2}-r_{+}^{n}-k l^{2} \delta_{n, 2}+\frac{3 k^{2}}{4} l^{4} \delta_{n, 4}\right. \\
& \left.-\frac{5 k}{8} l^{6} \delta_{n, 6}+\cdots\right),
\end{aligned}
$$

where again $\sigma_{k, n-1}$ is the (dimensionless) volume associated with the unit metric $d \Sigma_{k, n-1}^{2} / l^{2}$. Using Eq. (32) we can compute the energy and entropy of the solutions by application of standard thermodynamical formulas. One finds

$$
E=\frac{(n-1) \sigma_{k, n-1}}{16 \pi G} \mu+E_{k}^{0},
$$

where we denote by

$$
E_{k}^{0}=\frac{\sigma_{k, n-1}}{16 \pi G}\left(-k \delta_{n, 2}+\frac{3 k^{2}}{4} l^{2} \delta_{n, 4}-\frac{5 k}{8} l^{4} \delta_{n, 6}+\cdots\right)
$$

the terms that are independent of the black hole parameters (e.g., of the temperature). Their contribution to the action is therefore of the form $\beta E_{k}^{0}$. Note that one can extrapolate this Casimir energy to

$$
E_{k}^{0}=\frac{\sigma_{k, n-1}}{8 \pi G}(-k)^{n / 2} \frac{(n-1) ! !^{2}}{n !} l^{n-2},
$$

for arbitrary even $n$ using Eq. (21).

The entropy

$$
S=\frac{\sigma_{k, n-1} r_{+}^{n-1}}{4 G}
$$

satisfies the area law, and is independent of the extra terms $\beta E_{k}^{0}$. Not surprisingly, the result is therefore the same as in a background calculation.

Curiously, the results for $n=3$ and $n=4$ show different qualitative features. For $n=3$ the result that we obtain is the same as one would obtain by performing a background subtraction from the locally $\mathrm{AdS}_{4}$ solution neglecting the conical singularity that would appear for $k=-1$. This is rather similar to what we will find for TN-AdS in the next section: the method of counterterm subtraction appears to reproduce the results of an "imperfect matching" calculation. As a 
result, the extremal solution (29) has negative energy, whereas the locally AdS solution, with $\mu=0$, has vanishing action and energy.

By contrast, the result for the hyperbolic $n=4$ black holes supports the opposite scenario. The action (32) in this case reproduces precisely that obtained by taking the extremal state (30) as the reference state, and not the locally AdS state [notice that $I=0$ for the values in Eq. (30)]. For $n=4$ and $k=-1$, the energy (33) of the extremal state vanishes, a confirmation that this is to be taken as the ground state of the theory. The term $E_{k}^{0}$ is independent of the black hole parameters (e.g. the temperature), and its contribution to the action is therefore simply of the form $\beta E_{k}^{0}$.

For $k=1$ this term has been identified in Ref. [18] as precisely the Casimir energy associated to $\mathcal{N}=4$ superYang-Mills theory on the static Einstein spacetime $R \times S^{3}$, which is the spacetime obtained as the boundary of $\operatorname{AdS}$ spacetime in this case. This agreement is a striking outcome of the counterterm subtraction method. Notice that the interpretation as a Casimir energy is the only possible one, given that the AdS solution is the one with the lowest action and energy among that family-i.e., it is the ground state.

We would like to see whether a similar correspondence holds for $k=-1$. In this case it is crucial to notice that the ground state is not the locally AdS solution. The latter should be regarded as an excited state of the system. The ground state is the extremal solution, which has zero energy. By translating this into the AdS-CFT correspondence we would not expect to find a Casimir energy for the field theory calculations on the open static universe $\mathbb{R} \times H^{3}$. Indeed, the effective action and renormalized stress-energy tensor for conformal fields vanish on that space (see, e.g., Ref. [28]). This is in perfect agreement with the zero energy results that we find for the ground state (30) of the theory.

There are, however, some aspects that are in need of further exploration. In particular, from the entropy formula we see that for $k=-1$, not only does the locally AdS solution have non-zero entropy, but so does the extremal ground state. In particular, for $n=4$,

$$
S_{\mathrm{ext}}=\frac{\sigma_{k=-1,3} l^{3}}{2^{7 / 2} G} .
$$

In this respect, this ground state bears resemblance to the extremal black hole ground state discussed in [32], which had non-vanishing entropy as well. It is of great interest to understand this result (37) from a field-theoretical point of view. The "precursor"' states of Ref. [33] — constructed in standard field theory-might be extremely relevant to such a discussion. As proposed in Ref. [33], these are degrees of freedom that do not contribute to the energy density, although they store information. This looks precisely like what is needed to account for an entropy like we have found in Eq. (37). Perhaps the entropy of this ground state and the one presented in Ref. [32] represents the count of the number of precursor degrees of freedom in the field theory.

For black holes in $\mathrm{AdS}_{6}$ (i.e., $n=5$, and in fact, all odd values of $n$ ) the conclusions are essentially the same as in
$\mathrm{AdS}_{4}$. However, the situation for $\mathrm{AdS}_{7}$ is somewhat enigmatic. In this case, the action does not vanish either for the extremal black hole or for the locally AdS solution. Also, the energy is non-zero for both. Perhaps this is consistent with yet to be understood properties of the $(2,0)$ superconformal field theory that resides on the world volume of the M5brane [37].

Finally, it is of interest to note that because the "small" Schwarzschild black holes (in the sense of Ref. [7]) survive the $l \rightarrow \infty$ limit, (i.e., the cosmological constant goes to zero), the surface counterterm subtraction method supplies results for the action, energy and entropy for ordinary Schwarzschild black holes. For odd $n$, these results coincide precisely with those obtained by the background subtraction method, using Minkowski spacetime as a reference. For even $n$, the results would again coincide with the standard results in asymptotically flat space, except for the constant contribution of the Casimir energy (35) (and the analogous term in the action). In this case because for $n \geqslant 4$ this energy is proportional to $l^{n-2}$, it becomes an infinite constant in the limit $l$ $\rightarrow \infty$. We will see that this ability to take the flat spacetime limit occurs for other interesting solutions in the next section, and allows us to address and resolve certain situations which were fraught with uncertainties and/or ambiguities in the literature.

\section{ANTI-de SITTER NUTCRACKER}

As we mentioned in the Introduction, the issue of choosing a correct reference state for background subtraction has been a matter of some controversy for Taub-NUT and Taubbolt solutions, in the asymptotically locally flat situation $[25,27]$ as well as in the asymptotically locally AdS case $[14,15]$.

Note that in this section $n$ will be used to denote the "nut charge," not the number of dimensions-we will only deal with four-dimensional solutions.

\section{A. Spherical nuts and bolts}

The Taub-NUT-anti-de Sitter (TN-AdS) solution is

$$
\begin{aligned}
d s^{2}= & V(r)(d \tau+2 n \cos \theta d \varphi)^{2}+\frac{d r^{2}}{V(r)} \\
& +\left(r^{2}-n^{2}\right)\left(d \theta^{2}+\sin ^{2} \theta d \varphi^{2}\right),
\end{aligned}
$$

where

$$
V=\frac{\left(r^{2}+n^{2}\right)-2 m r+l^{-2}\left(r^{4}-6 n^{2} r^{2}-3 n^{4}\right)}{r^{2}-n^{2}} .
$$

Here we will simply sketch some of the features of the solution. For a detailed analysis we refer the reader to Ref. [14]. If $n=0$, we recover the Schwarzschild-AdS solutions with $m$ as a mass parameter. The analytically continued time, $\tau$, parametrizes a circle, $S^{1}$, which is fibered over the two sphere $S^{2}$, with coordinates $\theta$ and $\varphi$. The non-trivial fibration is a result of a non-vanishing "nut charge" $n$. As a 
result, the boundary as $r \rightarrow \infty$ is described as a "squashed" three-sphere, where $4 n^{2} / l^{2}$ parametrizes the squashing.

Euclidean regularity of the solution restricts the period of $\tau$ to be

$$
\beta=8 \pi n .
$$

In addition, the mass parameter has to be restricted so that the fixed point set of the Killing vector $\partial_{\tau}$ at radial position $r=r_{+}$is a regular one. Hence one finds "nut" or "bolt" solutions, depending on whether the fixed point set is zero or two dimensional, respectively. In particular, for "nut" solutions

$$
r_{+}=n, \quad m_{n}=n-\frac{4 n^{3}}{l^{2}} .
$$

In what follows, by TN-AdS we will mean the Taub-NUTAdS solutions with this particular value of $m$. Notice that $m_{n}$ vanishes for the value $n=l / 2$. It was shown in Ref. [14] that for this particular value the solution is precisely $\mathrm{AdS}_{4}$, with the slicing in which the sections at constant $r$ are round three-spheres. In contrast, the solution with $n=m=0$ corresponds to $\mathrm{AdS}_{4}$ with slices of geometry $S^{1} \times S^{2}$. For Taubbolt-AdS (TB-AdS) the expressions are more complicated [14]:

$$
\begin{gathered}
m_{b}=\frac{r_{b}^{2}+n^{2}}{2 r_{b}}+\frac{1}{2 l^{2}}\left(r_{b}^{3}-6 n^{2} r_{b}-3 \frac{n^{4}}{r_{b}}\right), \\
r_{+}=r_{b \pm}=\frac{l^{2}}{12 n}\left(1 \pm \sqrt{1-48 \frac{n^{2}}{l^{2}}+144 \frac{n^{4}}{l^{4}}}\right) .
\end{gathered}
$$

For $r_{b}$ to be real the discriminant must be non-negative. Furthermore, we must take the part of the solution which corresponds to $r_{b}>n$. This gives

$$
n \leqslant\left(\frac{1}{6}-\sqrt{\frac{3}{12}}\right)^{1 / 2} l
$$

It is only for this range of parameters that one can construct real Euclidean TB-AdS solutions. Notice, in particular, that the AdS value $l=2 n$ lies outside this range.

In Refs. [14,15], the action of the TB-AdS solutions was computed by matching the solutions to a TN-AdS solution with the same value of the nut charge. The thermodynamics of TB-AdS solutions were then found to be rather similar to that of Schwarzschild-AdS black holes. However, this method precluded an analysis of the TN-AdS solutions by themselves, since they acted as reference states. A completely rigorous calculation of the action of TN-AdS could not be performed using the reference background method, simply because it is not possible to match pure AdS (the intuitively obvious candidate background) to TN-AdS, as they have incompatible slices for all $n$ except $n=l / 2$. Equipped with the counterterm subtraction procedure, we can now compute the action for TN-AdS, without any reference to a background.

With

$$
\sqrt{h}=\sqrt{V(r)}\left(r^{2}-n^{2}\right) \sin \theta, \quad \mathcal{R}=\frac{2}{r^{2}-n^{2}}-\frac{2 n^{2}}{\left(r^{2}-n^{2}\right)^{2}} V(r),
$$

we find, for a solution with generic values of $m$ and $n$,

$$
I=\frac{4 \pi n}{G l^{2}}\left(l^{2} m+3 n^{2} r_{+}-r_{+}^{3}\right)
$$

where, as we said above, $r_{+}$is the minimum possible value of $r$, where there is a fixed point of the Killing vector $\partial_{\tau}$. Of course, as explained above, Euclidean regularity demands either $m=m_{n}$ or $m=m_{b}$.

There are several things to note about this result. The first is a consistency check: if we subtract the values we obtain for the TB-AdS and TN-AdS solutions, $I_{\text {bolt }}-I_{\text {nut }}$, we recover (after some algebra) the result obtained in Refs. $[14,15]$ for the action of TB-AdS with TN-AdS as a reference. Of course this consistency is to be expected in general. The standard background subtraction requires the asymptotic geometry of the solution and its reference state match. Hence the counterterms which depend only on the intrinsic boundary geometry must be equal, and will cancel if one takes the difference of the counterterm subtracted actions.

Next, in the flat space limit $l \rightarrow \infty$ we obtain

$$
I \rightarrow \frac{4 \pi n m}{G}
$$

In particular, in this limit we find

$$
I_{\text {nut }} \rightarrow \frac{4 \pi n^{2}}{G}, \quad I_{\text {bolt }} \rightarrow \frac{5 \pi n^{2}}{G}
$$

These are precisely the results that were obtained in Ref. [25] by an "imperfect match", of the Taub-NUT solution to Euclidean Minkowski space. Indeed, the same "imperfect match" to AdS can be seen to reproduce the result (46) above. Even if it is not possible to match the squashed $S^{3}$ at the boundary to the boundary of $\mathrm{AdS}_{4}$ with the slicing $S^{1}$ $\times S^{2}$, a finite result can nevertheless be obtained by neglecting the non-trivial fibering and performing a standard background subtraction. Proceeding this way the bulk (volume) term yields, at large $r$,

$$
I_{\mathrm{bulk}}=\frac{4 \pi n}{G l^{2}}\left(l^{2} m+3 n^{2} r_{+}-r_{+}^{3}\right)+\frac{\pi n^{3} r}{G l^{2}}+O(1 / r) .
$$

In contrast to other action calculations in AdS spacetime, the bulk term, even after subtraction, is not finite by itself; rather one needs to take into account the Gibbons-Hawking boundary term:

$$
I_{\text {surf }}=-\frac{1}{8 \pi G} \int_{\partial \mathcal{M}} d^{3} x \sqrt{h}\left(K-K_{0}\right)=-\frac{\pi n^{3} r}{G l^{2}}+O(1 / r) .
$$


By adding Eq. (49) and (50) and taking $r \rightarrow \infty$ we therefore recover Eq. (46).

We therefore conclude that the fact that the match to the background is an imperfect one does not appear to be as bad as it looks at first sight. Certainly, the result (47) of Ref. [25] in the ALF limit is on a better standing after having recovered it from a counterterm calculation.

Now we return to the result (46), and specialize to nut solutions using Eq. (41):

$$
I_{\mathrm{TN}-\mathrm{AdS}}=\frac{4 \pi n^{2}}{G}\left(1-\frac{2 n^{2}}{l^{2}}\right) .
$$

For $n=l / 2$ we recover the value for $\mathrm{AdS}_{4}$ with boundary $S^{3}$, which will be obtained and discussed in Sec. VI, whereas for $n=0$ we recover the value (zero) for $\mathrm{AdS}_{4}$ with boundary $S^{1} \times S^{2}$. Again, these special cases may be regarded as consistency checks on the internal consistency of our implementation of the procedure.

Notice that the action becomes negative for $n>n_{0}$ $=l / \sqrt{2}$. More interestingly, being able to vary the value of the Euclidean period $\beta=8 \pi n$ we can compute the energy of the solutions,

$$
E=\partial_{\beta} I=\frac{m_{n}}{G},
$$

which confirms the interpretation of $m$ as a mass parameter. We may go further and compute the entropy and specific heat:

$$
\begin{gathered}
S=\partial_{\beta} I-I=\frac{4 \pi n^{2}}{G}\left(1-\frac{6 n^{2}}{l^{2}}\right), \\
C=-\beta \partial_{\beta} S=\frac{8 \pi n^{2}}{G}\left(-1+12 \frac{n^{2}}{l^{2}}\right) .
\end{gathered}
$$

As had been already noticed in Ref. [14], the mass (energy) becomes negative for $n>l / 2$. More strikingly, the entropy becomes negative for $n>l / \sqrt{6}$. In particular, the entropy of $\mathrm{AdS}_{4}(n=l / 2)$ is negative (equal to minus its action, since it has $E=0)$. Whereas a negative mass may not be too troublesome (one may shift the energy scale), a negative entropy certainly would appear to be a sign of pathological behavior. One should keep in mind, however, that this negative entropy appears because of a particular choice of (Euclidean) time coordinate. Even if it may seem surprising at first sight that $\mathrm{AdS}_{4}$ suffers from this pathology, we stress that this is a consequence of the particular choice of time slicing that we have made here, rather than an instrinsic property of the $\mathrm{AdS}_{4}$ solution itself.

In Ref. [34] it was pointed out that in spaces where Euclidean time is non-trivially fibered there appeared a contribution to the entropy other than the usual one coming from the bolts (the latter yields the black hole area law). This extra entropy can be associated to "Misner strings" [35] (a geometric analogue of Dirac strings), and we would expect it to contribute to the entropy of TN-AdS as $S_{\mathrm{MS}}=A_{\mathrm{MS}} /(4 G)$ $-\beta H_{\mathrm{MS}}$, [36] where $A_{\mathrm{MS}}$ is the area of the string and $H_{\mathrm{MS}}$ is the Hamiltonian on it. Indeed, in the absence of a bolt this appears to be the only possible source of gravitational entropy for the TN-AdS solution. A brief calculation confirms that $S_{\mathrm{MS}}$ corresponds precisely to the expression we obtained in Eq. (53).

The fact that the specific heat becomes negative for $n$ $<l / \sqrt{12}$ is an indication that the solutions become thermally unstable, making them unusable for equilibrium thermodynamics ${ }^{8}$ (in the canonical ensemble). So if we declare that the physically relevant solutions are those with both positive entropy and positive specific heat, then the valid range for the nut charge is

$$
\frac{l}{\sqrt{12}} \leqslant n \leqslant \frac{l}{\sqrt{6}}
$$

Solutions in this range have positive action and positive energy.

Finally, we note that the results for the energy, entropy and specific heat of TB-AdS can be recovered by combining those for TN-AdS above, and those for TB-AdS with the TN-AdS subtraction in Ref. [14].

\section{B. Remarks upon field theory on squashed three-spheres}

As discussed in Ref. [14,15], the study of solutions with nut charge which are locally asymptotically AdS is relevant to the $2+1$ dimensional "exotic" [38] conformal field theories which reside on the world volume of M2-branes (and closely related theories ${ }^{9}$ ), after placing them on squashed three-spheres. Following that work, in Ref. [39] the effective actions of various fields on squashed three-spheres have been computed.

We do not expect to see in those particular field theory results any signal of the apparently pathological behavior (e.g., negative entropy) which we have found, and indeed we do not. The difficulty essentially lies in the fact that the field theory results can only be used at weak coupling, whereas supergravity is describing a strongly coupled regime of the field theory. The unusual behavior belongs only to the low temperature phase of the field theory, and strong coupling effects change the picture drastically. Recall the phase structure described in Ref. [14]:

(i) At high $T$ (small $n$ ) we have both TN-AdS and TBAdS as possible solutions, but the latter has the lower free energy, and is therefore preferred. It was shown in Ref. [14] that at high $T$, TB-AdS gives the expected behavior $F \sim T^{3}$ which, not surprisingly, is the result found in Ref. [39]. This is a deconfined phase.

\footnotetext{
${ }^{8}$ Nevertheless, a negative specific heat is not so bad as a negative entropy; as a matter of fact, as is well known, the Schwarzschild black hole in asymptotically flat spacetime has negative specific heat-and so does the ALF Taub-NUT solution.

${ }^{9}$ Recall there is a problem with the spin structure of TB-AdS, and so the M-theory interpretation is unclear [14], although there is almost certainly a dual CFT nonetheless.
} 
(ii) At low $T$ (large $n$ ), however, the only existing phase is TN-AdS. There is a phase transition separating this regime from the deconfined phase mentioned above. This phase transition prevents us from obtaining information from the results in Ref. [39], since at weak coupling, where those results were obtained, one does not get the confined phases.

It is in this large $n$ region that the entropy becomes negative. In fact, all of the negative entropy regime is within the region where the only regular solution is TN-AdS: TB-AdS is absent there. One might speculate whether the Lorentzian version of the field theory (in this confined phase) contains ghosts that do not decouple. Such ghosts would yield a negative contribution to the entropy.

So we discover that the supergravity studies give us new information on the strongly coupled phases of the theory on the world volume of the M2-brane and related theories after compactification on squashed three-spheres.

\section{Flat and hyperbolic Taub-NUT-AdS}

A solution where the nuts and bolts are flat planes instead of spheres can be found as well, and was analyzed in Ref. [14],

$$
\begin{aligned}
d s^{2}= & V(r)\left(d \tau+\frac{n}{l^{2}}(x d y-y d x)\right)^{2}+\frac{d r^{2}}{V(r)}+\frac{r^{2}-n^{2}}{l^{2}} \\
& \times\left(d x^{2}+d y^{2}\right),
\end{aligned}
$$

where, now,

$$
V=\frac{-2 m r+l^{-2}\left(r^{4}-6 n^{2} r^{2}-3 n^{4}\right)}{r^{2}-n^{2}} .
$$

The fibration is in this case a trivial one, and as a result the Euclidean period $\beta$ is independent of $n$. Zero dimensional fixed point sets of $\partial_{\tau}$ ("nuts") exist for $m_{n}=-4 n^{3} / l^{2}$. Solutions with bolts have a higher value of $m$. The result for the counterterm calculation of the action for a solution with generic $m$ and $n$ is

$$
I=\frac{\beta L^{2}}{8 \pi G l^{2}}\left(m l^{2}-r_{+}^{3}+3 n^{2} r_{+}\right),
$$

where, as usual, $r_{+}$is the radial position of the fixed point set ( $r_{+}=n$ for a nut), and $L^{2}$ accounts for the area of the $(x, y)$ plane, $-L / 2 \leqslant\{x, y\} \leqslant L / 2$. It can be easily checked that the action of Ref. [14], where the nut solution was taken as a reference background, can be recovered from Eq. (58) as $I$ (bolt) $-I$ (nut). Moreover, Eq. (58) is the same result we would obtain had we performed a background subtraction calculation with "imperfect matching" to $\mathrm{AdS}_{4}$ [the latter in its flat incarnation as $n=m=0$ in Eq. (56)]. We note that for the nut values the action is negative, which reflects the fact that its energy is negative-its entropy vanishes, as could have been expected in the absence of bolts or Misner strings, so in fact we find $I_{\text {nut }}=\beta E_{\text {nut }}$.

The last possibility is that of having hyperbolic fixed point sets of $\partial_{\tau}$. The explicit solution is

$$
\begin{aligned}
d s^{2}= & V(r)[d \tau+2 n(\cosh \theta-1) d \varphi]^{2}+\frac{d r^{2}}{V(r)}+\left(r^{2}-n^{2}\right) \\
& \times\left(d \theta^{2}+\sinh ^{2} \theta d \varphi^{2}\right),
\end{aligned}
$$

with

$$
V=\frac{-\left(r^{2}+n^{2}\right)-2 m r+l^{-2}\left(r^{4}-6 n^{2} r^{2}-3 n^{4}\right)}{r^{2}-n^{2}} .
$$

The fibration is trivial, and again, there are no Misner strings. However, it was found in Ref. [14] that there are no hyperbolic nuts: i.e., it is not possible to make $r=n$ into a regular fixed point of $\partial_{\tau}$. Nevertheless, bolt solutions can be constructed. This is rather analogous to the situation we encounter for hyperbolic black holes in Sec. IV. The result for the action is again formally very similar to Eqs. (46) and (58),

$$
I=\frac{\beta \sigma}{8 \pi G l^{2}}\left(l^{2} m+3 n^{2} r_{+}-r_{+}^{3}\right),
$$

where $\sigma$ is the area of the hyperbolic space [if quotients of $H^{2}$ are taken to yield surfaces of genus $g>1$ (this is not essential) then $\sigma=4 \pi(g-1)]$.

\section{AdS REVISITED}

Many of the quantities we have been computing can be translated into field theory results by using the dictionary provided by the AdS-CFT correspondence $[2,40]$, namely,

$$
\begin{aligned}
c & =\frac{3 l}{2 G} \quad \text { for } \mathrm{AdS}_{3}, \quad N^{3 / 2} \approx \frac{l^{2}}{G} \quad \text { for } \mathrm{AdS}_{4}, \\
N^{2} & =\frac{\pi l^{3}}{2 G} \quad \text { for } \mathrm{AdS}_{5}, \quad N^{3} \approx \frac{l^{5}}{G} \quad \text { for } \mathrm{AdS}_{7},
\end{aligned}
$$

where $c$ is the central charge of the dual CFT in two dimensions. The powers of $N$ displayed above are measures of the number of "unconfined" degrees of freedom: for $\mathrm{AdS}_{5}, N$ is the rank of the gauge group of the dual $\mathcal{N}=4$ supersymmetric four dimensional $S U(N)$ Yang-Mills theory. Meanwhile, for $\mathrm{AdS}_{4}$ and $\mathrm{AdS}_{7}$, the dual field theories are the ones $[37,38]$ that describe the world-volume dynamics of $N$ parallel M2-branes, and M5-branes, respectively. The details of these latter two theories are still rather indirectly and poorly understood, and the precise numerical relationship between factors [missing in Eq. (62) for these cases] will not be needed here, as we will make no precise numerical comparison. While there is almost certainly a dual conformal field theory for the case of $\mathrm{AdS}_{6}$, we will not comment upon it further. Note again that AdS for all of the dimensions listed are cases that can be handled with the counterterms that we now have.

In Sec. III, we considered the counterterm action for AdS with the boundary geometries $S^{1} \times M_{k}^{n-1}$. In those cases, the action is finite and interestingly for even $n$, an extra contribution appears of the form $\beta E_{k}^{0}$ where $E_{k}^{0}$ is a constant energy - see Eq. (35) in Sec. IV. This constant energy is 
readily interpreted in the dual field theory as a Casimir energy of the conformal field theory on $S^{1} \times M_{k}^{n-1}$ - see Ref. [42] for another discussion of Casimir energies in the AdSCFT correspondence. We can consider these results for $n$ $=2,4$ in more detail: the well known Casimir energy of ( 1 +1 ) dimensional CFT when going from the infinite plane to the cylinder $\mathbb{R} \times S^{1}$ is reproduced by the term $n=2$ in Eq. (35). Similarly, the Casimir energy of four dimensional Yang-Mills theory on $\mathrm{R} \times S^{3}$ is precisely the value of $E_{k=+1}^{0}$ for $n=4$ [18].

We found there as well that for the theory on $\mathbb{R} \times H^{3}$, even if $E_{k=-1}^{0} \neq 0$, the result is consistent with the absence of a Casimir energy after identifying correctly the ground state of the theory. We remarked as well upon the striking appearance of a non-zero entropy for this ground state, which strongly suggests the presence of degrees of freedom which can contribute to the entropy but not to the energy density, just like the "precursor" states identified in Ref. [33]. (This also reminds us of the non-zero entropy extremal ground state studied in Ref. [32].)

We can translate some of our results for the cases of $\mathrm{AdS}_{4}$ and $\mathrm{AdS}_{7}$ as well, finding that the Casimir energies derived by using Eq. (62) are correctly proportional to the number of degrees of freedom in the theory, as can be deduced from the power of $N$ which appears in each case: The scaling with $N$ is precisely the same as had been obtained from computations of black brane entropies [41].

Let us now consider $\operatorname{AdS}_{n+1}$ with boundary geometries $S^{n}$ and $H^{n}$ as described by the metrics in Eq. (7). In order to notationally distinguish them from the family $S^{1} \times M_{k}$, we will denote them with $a_{k}{ }_{k}$. The results for the action are somewhat more complicated to express for generic $n$ in an explicit form. For the three contributions (the bulk term, the Gibbons-Hawking surface term, and the counterterm action) we find

$$
I_{\text {bulk }}^{\bullet}=\frac{n \sigma_{k, n}}{8 \pi G l} \int_{r_{+}}^{r} d \bar{r} \frac{\bar{r}^{n}}{\sqrt{\bar{r}^{2}+k l^{2}}},
$$

which can be expressed in terms of hypergeometric functions, but we will only need its expansion for large $r$. The lower integration limit is $r_{+}=0$ for $k=+1,0$, and $r_{+}=l$ for $k=-1$ :

$$
\begin{aligned}
& I_{\text {surf }}^{\bullet}=-\frac{n \sigma_{k, n}}{8 \pi G l} r^{n} \sqrt{1+k \frac{l^{2}}{r^{2}}}, \\
& I_{\mathrm{ct}}^{\bullet 1}=\frac{(n-1) \sigma_{k, n}}{8 \pi G l} r^{n}, \\
& I_{\mathrm{ct}}^{\bullet 2}=\frac{(n-1) \sigma_{k, n}}{8 \pi G l} r^{n}\left(\frac{n}{2(n-2)} \frac{k l^{2}}{r^{2}}\right), \\
& I_{\mathrm{ct}}^{\bullet 3}=\frac{(n-1) \sigma_{k, n}}{8 \pi G l} r^{n}\left(-\frac{n}{8(n-4)} \frac{k^{2} l^{4}}{r^{4}}\right),
\end{aligned}
$$

where we have separated the contributions of the individual terms in the counterterm action (17), as was done in Eq. (18). Again the limit $r \rightarrow \infty$ remains to be taken. Our counterterms allow us to deal with $n=2, \ldots, 6$. We therefore find for $I_{k, n+1}^{\bullet}$

$$
\begin{gathered}
I_{k, 3}^{\bullet}=-k \frac{l \sigma_{k, 2}}{16 \pi G}\left(1+2 \log \frac{2 r}{l}\right), \\
I_{k, 5}^{\bullet}=k^{2} \frac{3 l^{3} \sigma_{k, 4}}{64 \pi G}\left(-1+4 \log \frac{2 r}{l}\right), \\
I_{k, 7}^{\bullet}=k \frac{5 l^{5} \sigma_{k, 6}}{64 \pi G}\left(\frac{5}{4}-3 \log \frac{2 r}{l}\right), \\
I_{k, 4}^{\bullet}=\frac{l^{2} \sigma_{k, 3}}{4 \pi G} \delta_{k,+1}, \quad I_{k, 6}^{\bullet}=0,
\end{gathered}
$$

where we have omitted contributions which vanish in the limit $r \rightarrow \infty$. Here the most striking result is that for even $n$, Eqs. (66), there remain logarithmically divergent contributions from the bulk terms that are not cancelled by the boundary counterterms. Furthermore, given their logarithmic nature, there is no way that they can be cancelled by a counterterm which is a local integral over the boundary of a (polynomial) curvature invariant. The appearance of these divergences then presents a limitation for the utility of the counterterm subtraction technique for investigations of asymptotically AdS solutions in odd dimensions. ${ }^{10}$

However, these divergences do not signal a problem for the AdS-CFT correspondence, but rather provide a remarkable consistency check. The possible existence of logarithmic divergences for odd spacetime dimensions was noted in Refs. [4,17], where the coefficients of the divergent terms were related to the conformal anomaly in the dual field theory. It is a standard result of field theory in curved spacetime $[28,43]$ that the appearance of a conformal anomaly in a classically conformally invariant theory is due to logarithmic UV divergences (at least at the one-loop level) appearing in the quantum field theory. Thus we have the UV-IR relation [45] of the AdS-CFT correspondence at work here: the appearance of an infinite volume singularity in the AdS calculation is a reflection of the existence of a UV divergence in the CFT.

Further, if we make the association of the AdS radius with an energy scale, we see that the divergence is logarithmic as required by the field theory. For $n=4$, it is straightforward to verify that in fact the $\mathcal{N}=4$ Super-Yang-Mills (SYM) theory has a conformal anomaly on $S^{4}$ or $H^{4}$, and further a perturbative weak coupling calculation reveals a logarithmic sin-

\footnotetext{
${ }^{10}$ One could consider the addition of nonpolynomial counterterms to resolve this problem. A suitable counterterm would have the form $a_{n / 2}(\mathcal{R}) \log f(\mathcal{R})$ where $a_{n / 2}(\mathcal{R})$ is the conformal anomaly term (see below) and $f(\mathcal{R})$ is an arbitrary curvature scalar. While such a counterterm would render the action finite, it may produce problematic results in calculating the boundary stress energy $[18,23]$. We would like to thank Sergey Solodukhin for this suggestion.
} 
gularity in the effective action for the background metric at oneloop $[43,44]$. That is, despite the remarkable finiteness properties of $\mathcal{N}=4 \mathrm{SYM}$ theory to higher loops in flat space [46], in curved spacetimes the $\mathcal{N}=4$ supersymmetry is only enough to protect against potential quadratic and linear divergences. In general though, there is the possibility of oneloop logarithmic divergences. One can show though that for the $\mathcal{N}=4$ SYM theory, the coefficient of these divergent terms will always vanish on product space geometries [44]. This is consistent with the fact that no logarithmic singularities were found in the actions (20) for the boundary geometries $S^{1} \times M_{k}$.

Let us make this connection somewhat more precise. In the presence of a trace anomaly term $T_{c}^{c}$ the action picks a divergent contribution of the form

$$
I_{\log }=\left(\log \frac{r}{l}\right) \int d^{n} x \sqrt{h} T_{c}^{c}
$$

[see, e.g., [17]. The cutoff $\epsilon$ in that paper is related to ours as $\left.\epsilon=(l / r)^{2}\right]$. Therefore we would expect, and we will actually verify it below, that the logarithmic terms we have found follow directly from the value of the anomaly.

Let us now write some of the results (66) in terms of field theory parameters, in order to make a comparison with the field-theoretical expression (68). The result for $n=4$ should be related to $\mathcal{N}=4$ supersymmetric four-dimensional YangMills theory on de Sitter $\left(S^{4}\right)$ or anti-de Sitter spacetime $\left(\mathrm{H}^{4}\right)$. We find a pleasingly simple result,

$$
I_{k, 5}^{\bullet}=-k^{2} \frac{N^{2}}{4}\left(1-4 \log \frac{2 r}{l}\right) .
$$

Note the fact that the action does not change sign when going from $S^{4}$ to $H^{4}$ (i.e., $k=+1 \rightarrow k=-1$ ) has its counterpart in the field theory in the fact that the divergent term in the effective action is given by curvature squared terms. In fact, this result generalizes to no sign change for $n=4 p$, where the conformal anomaly is proportional to the $2 p$ power of curvatures, and a change of sign for $n=4 p+2$, where the relevant power is $2 p+1$ [43].

Explicitly, for $\mathcal{N}=4$ SYM theory on $S^{4}$ the trace anomaly is [28]

$$
T_{c}^{c}=\frac{\sum_{s} q(s)}{240 \pi^{2} l^{4}}=\frac{3 N^{2}}{8 \pi^{2} l^{4}}
$$

where $q(s)$ measures the contribution of spin $s$ fields: for $\mathcal{N}=4$ SYM theory the result, for large $N$, is $\Sigma_{s} q(s)=90 N^{2}$.
Plugging this expression in Eq. (68) we recover the exact logarithmic term in Eq. (69).

The finite part of the action would be expected to follow from field-theoretical calculations as well. The scaling $N^{2}$ is just expected from the number of degrees of freedom of the theory, and the absence of any other factors follows from dimensional arguments. Related to this is the fact that the trace anomaly $\left\langle T_{c}^{c}\right\rangle$ can be computed exactly within the AdSCFT correspondence [17]. Having that, the full stress tensor follows in this case since the symmetry of the geometry will dictate that $\left\langle T_{a b}\right\rangle=h_{a b}\left\langle T_{c}^{c}\right\rangle / n$. Therefore, it is not surprising that a calculation of the stress tensor in the manner described in Ref. [18] reproduces this result.

For $\mathrm{AdS}_{3}$ we can write the result as

$$
I_{k, 3}^{\bullet}=-k \frac{c}{6}|g-1|\left(1+2 \log \frac{2 r}{l}\right),
$$

where $g$ is the genus of the two-dimensional boundary surface; i.e., for the hyperbolic case we have taken quotients by discrete groups in order to find genus $g$ surfaces (this is not essential). Again, the logarithmic term is precisely the result for a $(1+1)$ dimensional conformal field theory on a surface of genus $g$, area $4 \pi l^{2}|g-1|$, as follows from the trace anomaly on such a surface, $T_{b}^{b}=-k c /\left(12 \pi l^{2}\right)$.

In the same vein, we would expect that the presence of a logarithmically divergent factor for $\mathrm{AdS}_{7}$ can be interpreted in terms of the effective field theory for the M5-brane when defined on six dimensional de Sitter space. The anomaly for this theory has not been computed by independent fieldtheory methods; rather it has been deduced in Ref. [17] using the AdS-CFT correspondence. Using that result, the logarithmic term comes out precisely as expected.

It is clear that in the present paper we have only scratched the surface of the full subject, and more detailed and extensive comparisons between the results of Euclidean quantum gravity and the dual field theories are possible. We hope to report progress on this in the future.

\section{ACKNOWLEDGMENTS}

R.E. is supported by EPSRC through grant GR/L38158 (UK) and by grant UPV 063.310-EB187/98 (Spain). Support for C.V.J's research was provided by an NSF Career grant, No. PHY9733173. R.C.M's research was supported by NSERC (Canada) and Fonds FCAR du Québec. We would like to thank Vijay Balasubramanian, Cliff Burgess, Andrew Chamblin, Ivo Sachs and Joe Straley for comments and useful conversations.
[1] E. Witten, Adv. Theor. Math. Phys. 2, 253 (1998).

[2] J. Maldacena, Adv. Theor. Math. Phys. 2, 231 (1998).

[3] S.S. Gubser, I.R. Klebanov, and A.M. Polyakov, Phys. Lett. B 428, 105 (1998).

[4] E. Witten, Adv. Theor. Math. Phys. 2, 505 (1998).
[5] V. Balasubramanian, P. Kraus, and A. Lawrence, Phys. Rev. D 59, 046003 (1999); V. Balasubramanian, P. Kraus, A. Lawrence, and S.P. Trivedi, ibid. 59, 104021 (1999).

[6] G.W. Gibbons and S.W. Hawking, Phys. Rev. D 15, 2752 (1977); S. W. Hawking, in General Relativity, edited by. S. W. 
Hawking and W. Israel (Cambridge University Press, Cambridge, England, 1979).

[7] S.W. Hawking and D.N. Page, Commun. Math. Phys. 87, 577 (1983).

[8] S.W. Hawking and G.T. Horowitz, Class. Quantum Grav. 13, 1487 (1996).

[9] R.B. Mann, Class. Quantum Grav. 14, L109 (1997).

[10] D.R. Brill, J. Louko, and P. Peldan, Phys. Rev. D 56, 3600 (1997).

[11] L. Vanzo, Phys. Rev. D 56, 6475 (1997).

[12] R. Emparan, Phys. Lett. B 432, 74 (1998).

[13] D. Birmingham, Class. Quantum Grav. 16, 1197 (1999).

[14] A. Chamblin, R. Emparan, C.V. Johnson, and R.C. Myers, Phys. Rev. D 59, 064010 (1999).

[15] S.W. Hawking, C.J. Hunter, and D.N. Page, Phys. Rev. D 59, 044033 (1999).

[16] C. Fefferman and C.R. Graham, “Conformal Invariants,'” Elie Cartan et les Mathématiques d'aujourd'hui (Asterisque, 1985), p. 95.

[17] M. Henningson and K. Skenderis, J. High Energy Phys. 07, 023 (1998); hep-th/9812032.

[18] V. Balasubramanian and P. Kraus, hep-th/9902121.

[19] S. Hyun, W.T. Kim, and J. Lee, Phys. Rev. D 59, 084020 (1999).

[20] S.R. Lau, gr-qc/9903038.

[21] L.F. Abbott and S. Deser, Nucl. Phys. B195, 76 (1982).

[22] R. Arnowitt, S. Deser and C. Misner, in Gravitation: An Introduction to Current Research, edited by L. Witten (Wiley, New York, 1962).

[23] J.D. Brown and J.W. York, Phys. Rev. D 47, 1407 (1993).

[24] D. Kramer, E. Herlt, M. MacCallum and H. Stephani, in Exact Solutions of Einstein's Field Equations, edited by E. Schmutzer (Cambridge University Press, Cambridge, England, 1979).

[25] G.W. Gibbons and M.J. Perry, Phys. Rev. D 22, 313 (1980).

[26] J.P. Gauntlett, G.W. Gibbons, G. Papadopoulos, and P.K. Townsend, Nucl. Phys. B500, 133 (1997).
[27] C.J. Hunter, Phys. Rev. D 59, 024009 (1999).

[28] N.D. Birrell and P.C.W. Davies, Quantum Fields in Curved Space (Cambridge University Press, Cambridge, England, 1982).

[29] R.B. Mann, hep-th/9903229.

[30] R.M. Wald, General Relativity (The University of Chicago Press, Chicago, 1984).

[31] H. Liu and A.A. Tseytlin, Nucl. Phys. B533, 88 (1998).

[32] A. Chamblin, R. Emparan, C.V. Johnson, and R.C. Myers, Phys. Rev. D 60, 064018 (1999); (to be published), hep-th/9904197.

[33] J. Polchinski, L. Susskind, and N. Toumbas, hep-th/9903228.

[34] G.W. Gibbons and S.W. Hawking, Commun. Math. Phys. 66, 291 (1979).

[35] C. Misner, J. Math. Phys. 4, 924 (1963).

[36] S.W. Hawking and C.J. Hunter, Phys. Rev. D 59, 044025 (1999).

[37] E. Witten, hep-th/9507121; A. Strominger, Phys. Lett. B 383, 44 (1996).

[38] S. Sethi and L. Susskind, Phys. Lett. B 400, 265 (1997); T. Banks and N. Seiberg, Nucl. Phys. B497, 41 (1997).

[39] J.S. Dowker, Class. Quantum Grav. 16, 1937 (1999).

[40] J.D. Brown and M. Henneaux, Commun. Math. Phys. 104, 207 (1986).

[41] I.R. Klebanov and A.A. Tseytlin, Nucl. Phys. B475, 164 (1996).

[42] G.T. Horowitz and R.C. Myers, Phys. Rev. D 59, 026005 (1999); R. C. Myers, ibid. 60, 046002 (1999).

[43] See M.J. Duff, Class. Quantum Grav. 11, 1387 (1994), and references therein.

[44] C. Burgess, N.R. Constable, and R.C. Myers, J. High-Energy Phys. 08, 017 (1999).

[45] L. Susskind and E. Witten, hep-th/9805114.

[46] M. Grisaru, M. Rocek, and W. Siegel, Nucl. Phys. B183, 141 (1981); Phys. Rev. Lett. 45, 1063 (1980); S. Mandelstam, Nucl. Phys. B213, 149 (1983); L. Brink, O. Lindgren, and B.E.W. Nilsson, ibid. B212, 401 (1983). 